\title{
Actitudes autoritarias y violencia en Madrid
}

\author{
Florentino Moreno Martín ${ }^{1}$
}

RESUMEN Uno de los objetivos del Estudio Multicéntrico sobre Actitudes y Normas Culturales frente a la Violencia (proyecto ACTIVA) es analizar la relación entre las actitudes favorecedoras de la violencia y las conductas violentas. En el presente artículo se estudia la relación entre actitudes autoritarias y dos componentes de la violencia: su justificación y su realización efectiva. La recolección de datos se hizo en la ciudad de Madrid entre octubre y diciembre de 1996. Se trabajó con una muestra representativa de 1219 personas entrevistadas en sus domicilios a partir del cuestionario común del proyecto ACTIVA, al cual se añadieron algunas preguntas. Globalmente considerada, la muestra tuvo una puntuación baja en la escala de autoritarismo. El grupo de personas que más justificaron la violencia tuvo puntuaciones más altas en autoritarismo, al igual que las personas en cuyas conductas habituales se observó un nivel de agresión mayor. Las actitudes más autoritarias se constataron en barrios de bajos recursos económicos, en personas no activas laboralmente, en las de nivel educativo más bajo y en las que se identificaron con una ideología de derecha. Si los más autoritarios justifican y ejercen más la violencia, es preciso fomentar actitudes de crítica hacia quienes abusan del poder y respeto por las diferencias, a fin de prevenir la aparición de estas conductas, sin olvidar la influencia de las variables sociales mencionadas anteriormente.

Según los datos de la memoria de la Delegación del Gobierno en Madrid, en 1996 la tasa de homicidios en esta ciudad fue 2,15 por 100000 habitantes (1). Si se compara esta tasa con la de otras grandes ciudades latinoamericanas o europeas, se debería calificar el nivel de violencia de Madrid como bajo. No se percibe así entre los ciudadanos. Los incidentes entre pandillas de jóvenes en zonas de diversión, las riñas en los estadios de fútbol, las agresiones sexuales, los malos tratos dentro de la familia, los atracos y todo el rosario de sucesos que recogen los diarios cada día, aunque comparados con los vividos en otras ciudades no sean es-

\footnotetext{
Universidad Complutense de Madrid, Facultad de Psicología, Departamento de Psicología Social,. 28223 Madrid, España. Teléfono: 34.91.394.29.66, fax: 34.91.394.31.89, e-mail 1) fmoreno@correo. cop.es e-mail 2): pssoc12@emducms1. sis.ucm.es
}

pecialmente relevantes, son percibidos por los ciudadanos como si el nivel de violencia creciera sin parar. Este estado de opinión ciudadana ha llevado a la creación de una comisión especial del parlamento regional y a la financiación de varias investigaciones que pretenden desentrañar las formas de prevención y control de la violencia.

El fuerte impacto de los hechos violentos, especialmente de aquellos que derivan en consecuencias irreparables como la muerte, hace que la población se pregunte permanentemente cuáles son las causas de esas conductas. La mayor parte de los modelos explicativos, desde los más complejos hasta los unidireccionales, suelen tener poco éxito a la hora de convertirse en programas de intervención (2). Los primeros, porque al utilizar tantos factores explicativos y tantas y tan complejas relaciones entre los mismos, no resul- tan operativos, y los segundos, porque al atribuir la agresión a una única causa, que es tan inamovible (la dotación genética, por ejemplo) o tan difícil de evitar (como la frustración), apenas dejan margen de actuación.

Las viejas teorías sobre el condicionamiento genético de la conducta violenta $(3,4)$, a pesar de seguir siendo muy populares fuera de los círculos académicos, han ido perdiendo fuerza (5) y han dejado paso a explicaciones de carácter psicosocial en las cuales los procesos de aprendizaje se utilizan como fuente de explicación. Desde los años setenta, se han realizado cientos de experimentos de laboratorio (6) que parecen validar el postulado de que la violencia se aprende y que este proceso puede darse de forma presencial (directa) o vicariante (indirecta) (7). La consecuencia más directa de estos estudios ha sido la popularización de la 
idea de que basta con que se presente un modelo violento para que una conducta similar se lleve a cabo, hipótesis que ha supuesto poner en el centro del debate sobre la violencia a la industria audiovisual como principal generadora de modelos violentos (8).

Tan reduccionistas eran los postulados biologicistas como los que consideran que la acción violenta puede explicarse como mera repetición de situaciones presenciadas. Tan evidente es que sin modelos claros la acción pierde probabilidades de aparición (9), como que existe una capacidad biológica para la acción violenta. Pero entre esa potencialidad y la realización de actos violentos (presenciados o vividos previamente) median un conjunto de procesos que pueden frustrar la manifestación de esas conductas (por muy bien modeladas que estuvieran), que pueden facilitarlas o empujarnos de forma imperativa a realizarlas.

Esos procesos intermedios se van adquiriendo desde la infancia (10) y se van consolidando en capacidades para afrontar las situaciones conflictivas (aptitudes o habilidades) y en actitudes o disposiciones a la acción (con sus componentes emocionales, cognitivos y volitivos). Lo interesante de estas actitudes que median entre la situación conflictiva y el comportamiento violento es que no tienen por qué estar orientadas directamente a la justificación o realización de la acción violenta. Si ciudadanos pacíficos se sienten orgullosos de asesinar a sus enemigos cuando estalla una guerra (11), o estudiantes amables infligen descargas eléctricas a sus compañeros cuando un profesor se lo ordena (12), no es porque se les haya educado en actitudes y valores de exaltación de la violencia o del sadismo, sino porque se sienten forzados a actuar debido a su vinculación emocional con una nación, su necesidad de obedecer a la autoridad o cualquiera de las normas y valores interiorizados como imperativos en el proceso de conformación de la personalidad. A partir de esta disposición a la acción, el ambiente cultural o subcultural de la persona regula cuándo se debe ejercer la violencia y cómo hacerlo. El individuo se ve im- pelido a cumplir con unos papeles ya establecidos bajo la amenaza del aislamiento social y el sentimiento de culpa por no cumplir con las expectativas generadas (13). Si se puede demostrar la relación entre estos componentes actitudinales y la expresión de la violencia, se podría abrir una vía para la intervención.

En 1950, un grupo de profesores de la Universidad de Berkeley (14) publicó un estudio influyente cuyo eje central estaba constituido por la tesis según la cual existía un tipo de personalidad, definida como autoritaria, que justificaba y favorecía la aparición de regímenes tan violentos como el fascismo. La caracterización de este tipo de personalidad, o síndrome, se definía a partir de las puntuaciones en una escala denominada $\mathrm{F}$ que abarcaba distintos factores: convencionalismo, sumisión a la autoridad, hostilidad hacia los transgresores, pensamiento estereotipado, desprecio por lo tierno y sentimental, tendencia a la superstición y al fatalismo, obsesión por lo relativo al poder y al sexo y pesimismo sobre la condición humana. La publicación de este estudio de Adorno y colaboradores tuvo un gran impacto, fundamentalmente por su premisa básica: las actitudes, valores y convicciones sociales y políticas del individuo forman un patrón coherente, que expresa tendencias profundas de la personalidad. En los años posteriores, a pesar de las fundadas críticas metodológicas que recibió $(15,16)$, la escala $\mathrm{F}$ se utilizó en investigación (17) y se construyeron modelos alternativos a la misma como los de Eysenck (18), con sus famosas dimensiones radicalconservador, autoritario-democrático y los de Rokeach $(19,20)$, con su escala de dogmatismo. Todos estos modelos, de una $\mathrm{u}$ otra forma, situaban la naturaleza del problema (generalmente definido operativamente como prejuicio y disponibilidad a la agresión) en las dinámicas de la personalidad individual, tendiendo a olvidar o a dar poca importancia a la influencia de los factores socioculturales. No tardaron en aparecer estudios transculturales, como el de Pettigrew (21), que limitaban el alcance de las diferencias individuales y consideraban al racismo, al autoritarismo o al dogmatismo como fruto de la adaptación a las normas sociales dominantes en un tiempo determinado, no como problemas o disfunciones de la personalidad. Así se explicaría cómo en un momento determinado algunas configuraciones actitudinales prejuiciosas están muy presentes en la población y, sobre todo, cómo en períodos breves de tiempo se modifican de forma radical. Las ideas de normatividad e historicidad, sin restar fuerza a la vinculación de las variables personales con la conducta, ofrecen una visión más optimista de las posibilidades del cambio de configuraciones actitudinales como el autoritarismo o el racismo, las cuales no tendrían como única vía la transformación de los procesos de socialización en la familia o la terapia psicológica.

Partiendo de la idea original del grupo de Berkeley (existe una relación positiva entre violencia y autoritarismo), pero sin asumir la visión generalista del concepto de personalidad, sino una visión más aplicada al concepto de actitud (22), el propósito de este artículo es hacer un análisis detallado y cuantitativo de la relación entre estos grandes conceptos en la muestra del estudio de la ciudad de Madrid. También se pretende analizar cuáles son las variables sociodemográficas relacionadas con los valores autoritarios, especialmente aquellas que en psicología social se considera que influyen directamente en la elaboración de los prejuicios, como el nivel educativo, que para algunos autores puede ser la explicación del autoritarismo (23), la edad y la condición socioeconómica (tratados en el estudio original de Adorno) y el género.

En este estudio, además, se pretenden verificar tres hipótesis básicas: 1) quienes justifiquen de forma más acusada las distintas formas de violencia, puntuarán más alto en la escala de actitudes intolerantes o autoritarias, 2) los que ejerzan la violencia hacia sus hijos, cónyuges o no familiares puntuarán más alto en la escala de autoritarismo que los que no la ejerzan, y 3) se supone que existen determinadas características sociodemográficas que 
están relacionadas diferencialmente con la escala de autoritarismo.

\section{MATERIALES Y MÉTODOS}

El universo del estudio estuvo constituido por la población de 18 o más años de edad empadronada en la ciudad de Madrid. No se puso límite superior de edad salvo la incapacidad manifiesta del entrevistado. En julio de 1996 esta población era de 2404726 personas. Como referencia del tamaño de la muestra se consideró el prefijado por la Organización Panamericana de la Salud (1 200 personas), y la muestra final seleccionada (excluidos los cuestionarios que no cumplieron las normas de calidad) estuvo integrada por 1219 personas. Utilizando como referencia el supuesto más desfavorable $(P=0,5)$ y un nivel de confianza de $95,5 \%$ (K= 2 sigmas), el error de muestreo fue de $\pm 2,86 \%$.

Para seleccionar la muestra se utilizó el censo suministrado por el Ayuntamiento de Madrid (información del 17 de julio de 1996) con datos segregados según las distintas unidades administrativas (21 distritos, 128 barrios y 2309 secciones censales). Se trabajó siempre con la unidad censal como referencia. Como criterio común del proyecto ACTIVA se realizó una estratificación inicial, utilizando como base la condición socioeconómica (baja, media y alta) de las secciones censales. Para asignar a las personas a los estratos, se elaboró una escala socioeconómica (con valores de 0 a 100) a partir de una combinación ponderada de los datos objetivos que se informaba en el censo: desempleo, tasa de ocupación (frente a no activos), nivel de estudios, categoría profesional, valor del sueldo y vehículos matriculados (según su cilindrada). Una vez definidos los estratos, se hizo una asignación proporcional de las secciones a los mismos. La selección de los conglomerados (secciones) se hizo por un procedimiento aleatorio con probabilidad proporcional al tamaño de cada sección. Una vez seleccionadas las secciones, la elección de hogares se llevó a cabo por el procedimiento de rutas aleatorias (24) y la de las personas dentro del hogar, mediante un muestreo aleatorio simple.

La muestra por grupos de edad y su comparación con la población total de Madrid se describen en el cuadro 1 . Globalmente, se observa una sobrerrepresentación de las mujeres en la muestra de 6,32\% (53,98\% de la población frente a $60,3 \%$ en la muestra). En cuanto a los grupos de edad, están sobrerrepresentados los menores de 44 años, lo que supone que la media de edad de la muestra es 2 años menor que la de la población $(43,1$ frente a 45,1$)$. En resumen, se trata de una muestra razonablemente ajustada, aunque con ligero predominio de mujeres y personas jóvenes respecto de la población de la ciudad de Madrid.

Las entrevistas realizadas directamente en los hogares no son habituales en Madrid debido a la alta resistencia de los ciudadanos a abrir las puertas de sus viviendas, la cual ha sido provo- cada por la fuerte presión comercial y, sobre todo, por los incidentes de atracos profusamente ilustrados en los medios de comunicación. Para respetar el método de recolección de datos acordado en el proyecto ACTIVA, el trabajo de campo se diseñó cuidadosamente del siguiente modo: se seleccionó un grupo de entrevistadoras y entrevistadores universitarios, que reunían ciertas aptitudes y experiencias previas. Se procedió a capacitarlos de forma intensiva durante dos semanas, tarea que incluyó: manejo de la muestra (uso de mapas detallados, de la hoja de ruta, procedimientos de selección de las unidades de muestreo, etc.); aprendizaje del cuestionario (tipo y forma de las preguntas, dificultades de preguntas concretas, formas de registro de rechazos, etc.); técnicas persuasivas para conseguir el consentimiento de la familia (uso de identificación, indumentaria, forma de trato, explicación de la importancia del estudio, etc.), y resolución de dudas y organización para el seguimiento del trabajo. Antes de iniciar la recolección de datos, cada entrevistador tuvo que realizar varias entrevistas de prueba. Seguidamente, se desarrolló una campaña de prensa, en televisión y radio principalmente, para alertar sobre la presencia de entrevistadores de la Universidad Complutense en las siguientes semanas (se emitieron entrevistas simuladas, aparecieron entrevistadores en la calle, etc.). A partir de esta campaña, se inició el trabajo de campo (en octubre de 1996), realizando visitas iniciales a las casas seleccionadas. En ellas se pegaba un cartel en el

CUADRO 1. Distribución del universo y de la muestra por edad y sexo. Madrid, España, 1996

\begin{tabular}{|c|c|c|c|c|c|c|c|c|c|c|c|c|}
\hline \multirow[b]{2}{*}{ Edad (años) } & \multicolumn{6}{|c|}{ Universo } & \multicolumn{6}{|c|}{ Muestra } \\
\hline & Hombre & $(\%)$ & Mujer & $(\%)$ & Total & $(\%)$ & Hombre & $(\%)$ & Mujer & $(\%)$ & Total & $(\%)$ \\
\hline $18-29$ & 320944 & 50,84 & 310367 & 49,16 & 631311 & 26,25 & 156 & 43,70 & 201 & 56,30 & 357 & 29,41 \\
\hline $30-44$ & 272826 & 47,47 & 301887 & 52,53 & 574713 & 23,90 & 117 & 34,21 & 225 & 65,79 & 342 & 28,17 \\
\hline $45-59$ & 255695 & 45,91 & 301302 & 54,09 & 556997 & 23,16 & 77 & 32,49 & 160 & 67,51 & 237 & 19,52 \\
\hline $60-74$ & 196532 & 42,89 & 261727 & 57,11 & 458259 & 19,06 & 99 & 48,06 & 107 & 51,94 & 206 & 16,97 \\
\hline $75-94$ & 60561 & 33,01 & 122885 & 66,99 & 183446 & 7,63 & 32 & 44,44 & 40 & 55,56 & 72 & 5,93 \\
\hline No registrada & - & - & - & - & - & - & 3 & 33,34 & 2 & 66,66 & 5 & - \\
\hline Total & 1106558 & 46,02 & 1298168 & 53,98 & 2404726 & 100,00 & 484 & 39,70 & 735 & 60,30 & 1219 & 100,00 \\
\hline
\end{tabular}

Fuente: Ayuntamiento de Madrid. Censo de 1996. 
que se explicaba a los vecinos la próxima visita del entrevistador. Además, se introducía una carta en el buzón de la vivienda seleccionada en la cual se explicaban detalladamente el procedimiento de selección, el propósito del estudio, el nombre del entrevistador, la fecha tentativa de la visita y el teléfono de información de la universidad. Un día después se visitaba el hogar seleccionado y se procedía según lo previsto en la fase de formación. Los entrevistadores tenían un protocolo de actuación elaborado por la universidad, que incluía el procedimiento para dar explicaciones iniciales (el entrevistado podía negarse a contestar cualquier pregunta que desease, anonimato, confidencialidad, etc.), el proceso de realización y el de finalización de la entrevista.

El cuestionario utilizado para recoger los datos fue el acordado en el proyecto ACTIVA con algunas modificaciones para adaptarlo a Madrid. En el mismo se incluyeron preguntas sobre la definición ideológica (de izquierda o de derecha) y sobre la opinión acerca de la negociación con el grupo separatista vasco ETA.

Del conjunto de preguntas del cuestionario utilizado, a continuación se presentan las que se utilizaron para verificar las hipótesis formuladas anteriormente. Dichas preguntas se dividen en cuatro grupos: 1) actitudes autoritarias, 2) justificación de la violencia, 3) violencia ejercida y 4) variables sociodemográficas.

La definición operativa del primer concepto, actitudes autoritarias, se hizo formulando preguntas que permitieran detectar a personas que sobrevaloraran el principio de la autoridad (mostrando una adhesión acrítica a las acciones de los que detentan el poder) y que mostraran baja tolerancia de las diferencias y de las transgresiones. Las preguntas formuladas, que se exponen a continuación, tenían cinco respuestas posibles, que abarcaban desde el total desacuerdo al total acuerdo: 1) Cuando la policía persigue a criminales, estaría justificado que invadiera una casa sin orden judicial; 2) Si la policía considerara sospechosos a determinados jóvenes por su aspecto físico, estaría justificado que los detu- viera; 3) En algunos casos se justifica que la policía torture a los sospechosos para obtener información; 4) Considero justificada la pena de muerte para ciertos crímenes; 5) Los niños delincuentes deben ser recluidos en instituciones penales, 6) Sería conveniente que un barrio estuviera compuesto por personas a) de una misma clase social, b) de una misma religión, c) de un mismo origen étnico o raza o d) con las mismas ideas políticas; 7) Me parece bien que la gente pueda echar de su barrio a ciertos grupos de personas, y 8) La presencia militar en las calles es necesaria para controlar la violencia en el país.

En cuanto a la justificación de la violencia, los conjuntos de preguntas de tipo escalar, en las que se presentaban a los entrevistados comportamientos frecuentes ante los cuales debían situarse en una escala de cinco opciones, se agruparon desde el "total desacuerdo" al "total acuerdo" (en unos casos) y desde "me parece bien" a "me parece mal", pasando por "me parece mal pero lo entiendo". Dos preguntas hicieron referencia a la justificación de la violencia hacia los niños: 1) Para educar a los menores es necesario el castigo físico y 2) Existen situaciones en las cuales se justifica que un adulto le pegue a un niño/a que no es su hijo/a. La justificación de la violencia en la pareja comprendió cuatro preguntas: 1) Existen situaciones en las cuales se justifica que un hombre le dé una bofetada a la esposa; 2) Existen situaciones en las cuales se justifica que una mujer le dé una bofetada al esposo; 3) Si una mujer ha sido infiel a su marido, merece que él le pegue, y 4) Si un hombre ha sido infiel a su esposa merece que ella le pegue. Por su parte, la valoración de la justificación de la violencia contra otros por causas que afectan a la familia estuvo integrada por las siguientes cinco preguntas: 1) Una mujer tiene el derecho de agredir a otra mujer que le está quitando a su marido; 2) Un hombre tiene el derecho de agredir a otro hombre que le está quitando a su esposa; 3) Suponga que una persona hiere seriamente a alguien que le quitó el/la esposo/a. A usted. . . ; 4) Suponga que una persona mata a alguien que le ha violado a su hija/o. A usted. .. , y 5) Considero justificado que una persona mate para defender a su familia. Por último, las siguientes tres preguntas hicieron referencia al apartado de valoración de la justificación de la violencia contra otros por otras causas: 1) Si hay una persona que mantiene atemorizada a su comunidad y alguien la mata, a usted. . . ; 2) Si un grupo de personas se dedica a matar a individuos indeseables, a usted. .. , y 3) Considero justificado que una persona mate para defender su casa o propiedad.

En lo que atañe a los actos violentos cometidos por el entrevistado, se registraron sus respuestas sobre sus recientes comportamientos violentos contra sus hijos, su pareja y contra otros individuos. Se trataba de preguntas escalares que aludían a la frecuencia (desde nunca hasta muy frecuentemente) con que se adoptaron tales conductas contra niños (en el último mes), la pareja y otras personas (durante los últimos 12 meses). No se elaboró una única escala de violencia ejercida, puesto que se trata de submuestras diferentes: todos los sujetos en el caso de la violencia no familiar, pero solo una parte en el caso de los que tenían hijos o pareja.

Las conductas violentas dirigidas hacia un niño menor, bien fuese tutorizado o hijo propio (si había varios se elegía al azar uno de los niños o niñas), fueron: 1) ¿Tuvo que gritarle con rabia?; 2) ¿Tuvo que darle algún "cachete"? y 3) ¿Tuvo que pegarle un "tortazo" o con algún objeto como un cinturón, un palo, el puño? Por otra parte, las conductas violentas ejercidas hacia la pareja se evaluaron con las siguientes tres preguntas: 1) ¿Gritó con rabia a su pareja?; 2) ¿Le dio una bofetada a su pareja?, y 3) ¿Usted le pegó a su pareja con un objeto que pudo haberle lastimado? En el cuestionario se repetían estas mismas preguntas, aunque desde la perspectiva pasiva de la victimización (cuántas veces le gritó, abofeteó o pegó su pareja a usted). Por último, las conductas violentas dirigidas hacia otros (no familiares) se evaluaron por medio de las siguientes cuatro preguntas: 1) En medio de algún problema, ¿usted insultó a alguien que no era familiar 
suyo?; 2) ¿Amenazó con lastimar seriamente a alguien que no era familiar suyo?; 3) ¿Golpeó a alguien que no era familiar suyo?, y 4) ¿Se valió de alguien para que hiciera mal a una persona con quien había tenido problemas?

Por otra parte, se estudiaron las variables sociodemográficas que pueden influir en las actitudes autoritarias. A este respecto, se analizó la relación con el autoritarismo de las principales variables de tipo sociodemográfico que se extrajeron de la bibliografía sobre el tema: la edad, el sexo, el nivel académico, la condición socioeconómica y la situación laboral.

Los métodos de análisis utilizados para verificar las tres hipótesis centrales detalladas anteriormente fueron, primero, la construcción de la escala de actitudes autoritarias (en adelante, autoritarismo). En ella se incluyeron preguntas sobre la adhesión acrítica a las acciones de las autoridades (de la policía, del sistema legal, etc.), así como la falta de tolerancia de las diferencias (deseo de vivir con personas similares) y de las transgresiones (expulsar a "ciertas personas", recluir a menores infractores, etc). Una vez hechas las debidas correcciones en la dirección de las respuestas, para que los valores más altos indicaran alto autoritarismo, se elaboró la escala de autoritarismo utilizando la técnica de Likert (25). Para averiguar la calidad de la escala, se hizo un análisis de fiabilidad que incluyó la correlación conjunta de los ítemes. El valor final de la alfa de Chronbach fue de 0,81. Al ser excluido, ninguno de los ítemes elevaba significativamente el valor final de la escala.

En el cuestionario también se incluyó un conjunto de preguntas relacionadas con la opinión sobre las autoridades, que se utilizaron para probar que la escala de autoritarismo era congruente con actitudes cercanas. Concretamente, se analizó el grupo de juicios sobre la "eficacia atribuida a distintas instituciones en su labor de promoción de la seguridad ciudadana". A los entrevistados se les solicitó valorar (de "muy mala" a "muy buena") la labor de la policía, los jueces, el sistema penitenciario, la fiscalía, el ejército, el Defensor del Pueblo, los medios de comunicación y los políticos. Por medio de un análisis de la varianza de un solo factor, y con un nivel de confianza de $95,5 \%$, se observó que el valor medio en autoritarismo era significativamente mayor cuanto mejor se calificase la labor del sistema penitenciario y del ejército (las dos instituciones más asociadas con el ejercicio directo y punitivo de la autoridad). Siguiendo el mismo procedimiento de contraste con otros ítemes, se observó que quienes más puntuaban en autoritarismo percibían mayor preocupación de los gobernantes por lo que les pasaba, sentían que tenían poca influencia en los cambios del país, preferían dejar el sistema como estaba, y consideraban que una dictadura podría ser buena en determinadas circunstancias. En los tres casos el valor $P$ asociado con la $F$ de Snedecor fue menor de 0,01 . Para los propósitos de este estudio se consideró que con estas pruebas la calidad de la escala quedaba suficientemente probada.

El proceso de justificación de la violencia está muy ligado a los grupos a los que pertenecen los sujetos, a la supuesta causa que motiva la agresión y a las víctimas de la misma (26). Por esta razón, en segundo lugar se crearon subescalas de justificación de la violencia, agrupando los ítemes que se han mencionado anteriormente: justificación de la violencia hacia niños, hacia la pareja, hacia otros por causas familiares y hacia otros por otras causas. Se utilizó la misma técnica de Likert, según la cual el valor mínimo posible es 1 (nula justificación) y el máximo, 5 (justificación plena).

En tercer lugar, se construyeron escalas de conductas violentas ejercidas. Para ello, los ítemes se agruparon en función del objeto de la agresión (hijos, pareja y otros no familiares), utilizando la misma técnica que en el caso anterior.

En cuarto lugar, y para verificar la primera hipótesis (quienes justifiquen la violencia en todos los niveles tendrán puntuaciones más altas en autoritarismo), se procedió del siguiente modo: una vez creadas las variables de justificación de la violencia hacia niños, la pareja, otros por cuestiones familiares y otros por otras causas, se calculó la distribución de frecuencias de cada una de estas nuevas escalas. En función de la aparición de los distintos tipos de respuesta, los sujetos se agruparon en tres grupos de tamaño semejante, tomando como referencia los percentiles 33,3, y 66,6. Los valores de estas nuevas variables se definieron como 1 = justificación baja, 2 = media y 3 = alta. Con estas variables, consideradas independientes, se realizó un análisis de la varianza en el cual el autoritarismo fue la variable dependiente. La hipótesis nula (igualdad de las varianzas, es decir, ausencia de diferencias en autoritarismo entre quienes justifican más o menos la violencia) se rechazaría a partir de los valores $P$ asociados con el estadístico $F$ de Snedecor, una vez efectuada la prueba Levene de homogeneidad de varianzas. El nivel de confianza escogido fue de $95,5 \%$.

Posteriormente, y con objeto de verificar la segunda hipótesis (quienes ejercen la violencia hacia niños, la pareja y hacia no familiares tendrán mayor puntaje en autoritarismo que quienes no lo hagan), se procedió del siguiente modo: una vez elaboradas las variables mediante la técnica de Likert, se calculó la distribución de frecuencias de cada una de estas nuevas escalas y se observó que buena parte de los integrantes de la muestra nunca habían tenido conductas violentas. Considerando que se trataba de manifestaciones verbales de los entrevistados y no de datos empíricos constatables (en los que sí se podrían establecer claramente matices entre violencia ejercida baja, media y alta), se decidió dicotomizar los valores de estas variables dando el valor 1 en las tres escalas a quienes no habían ejercido ningún tipo de violencia y el valor 2 a los que habían desarrollado alguna conducta agresiva aunque no fuera grave (por ejemplo, gritar con rabia alguna vez). A partir de estas nuevas variables dicotomizadas, se analizó si existían diferencias estadísticamente significativas en la escala de autoritarismo entre quienes ejercían la violencia y los que no la ejercían. Para ello, se utilizó la prueba $t$ de 
Student para muestras independientes. Además del análisis de las tres escalas, se analizó por separado si existían diferencias en el autoritarismo atendiendo a cada uno de los ítemes de cada escala (por ejemplo, gritar, "cachete" y golpear con objetos) y agrupando los malos tratos en los que hubiera un componente de violencia física ("cachete" y golpes con objetos) y en los que hubiera solo violencia verbal (gritar).

Finalmente, para analizar la influencia de los factores sociodemográficos en la escala de autoritarismo (tercera hipótesis), se realizaron distintas pruebas estadísticas en función del tipo de escala (análisis de la varianza, prueba $t$ de Student). En todos los casos se adoptó un nivel de confianza de $95,5 \%$.

\section{RESULTADOS}

Si solo se consideraran como referencia los valores matemáticos entre los que se movían los tres grupos de escalas (1, mínimo; 3, medio; 5 máximo) y se tuviera en cuenta que el valor final de cada escala era la media de los valores de los ítemes, se podría afirmar, por extrapolación a partir de los resultados obtenidos en los integrantes de la muestra de este estudio, que los madrileños son poco autoritarios (media = $2,06)$, justifican poco la violencia hacia la infancia (media $=1,49$ ), hacia la pareja (media $=1,34)$, hacia otros por causas familiares (media $=2,09$ ) y hacia otros por otras causas (media $=1,80$ ). Del mismo modo, los madrileños afirman ejercer poco la violencia hacia los niños, la pareja o los no familiares. En todos los casos, el valor más repetido fue el mínimo (1) y la mediana nunca superó el valor 2 . Tras el dato general de un valor medio bajo en conductas violentas ejercidas, los porcentajes de agresión manifestada son preocupantes. Solo tomando en cuenta los casos más graves, se observó que $27,7 \%$ de quienes tenían hijos les dieron un "cachete" en el último mes y 2,74\% los golpearon de forma más severa (bofetón o golpes con un cinturón, etc.). El 2,36\% de los entrevistados afirmaron haber dado a su pareja por lo menos una bofetada en el último año y $0,15 \%$, la gol- pearon con un objeto que pudo haberla lastimado.

Por otro lado, las personas con puntuaciones más altas en la escala de autoritarismo justificaron más el ejercicio de la violencia. Como puede apreciarse en la figura 1, los valores de autoritarismo (que podían variar entre un mínimo de 1 y un máximo de 5) fueron más altos cuanto más se justificó la violencia, independientemente del grupo o causa a la que hicieran alusión. En los cuatro casos, las diferencias fueron estadísticamente significativas $(P<0,0005)$ y en el sentido previsto: el grupo de justificación alta de la violencia tenía valores medios en autoritarismo mayores que los del grupo de justificación media, y estos fueron más altos que los de justificación baja.

Como se puede observar en la figura 2 , las personas que ejercieron la violencia hacia niños, la pareja o los no familiares tuvieron puntajes más altos en la escala de autoritarismo. En la escala de violencia ejercida contra la pareja, con el ítem "gritó con rabia" (contestado afirmativamente por $63,3 \%$ de los entrevistados) no se pudo discriminar a las personas más o menos autoritarias (los valores que aparecen se refieren a los dos ítemes correspondientes a "dar bofetadas" y "golpear con objeto"). Del mismo modo, en la escala de violencia ejercida contra no familiares el ítem

FIGURA 1. Autoritarismo versus justificación de la violencia. Madrid, España, 1996

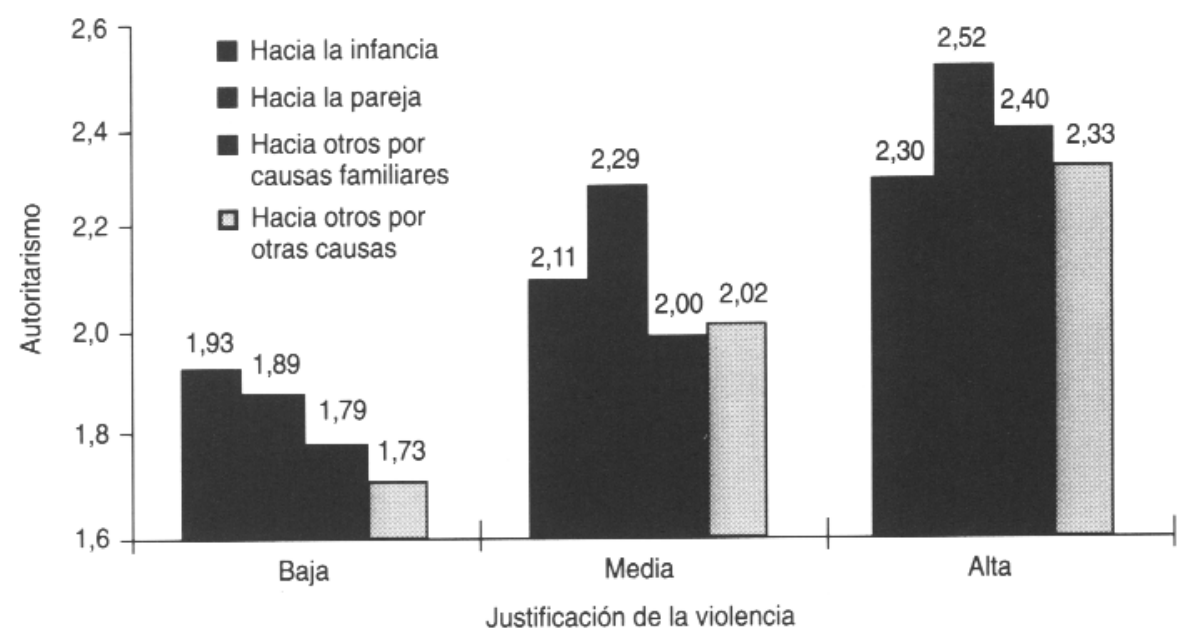

FIGURA 2. Autoritarismo versus conductas violentas. Madrid, España, 1996

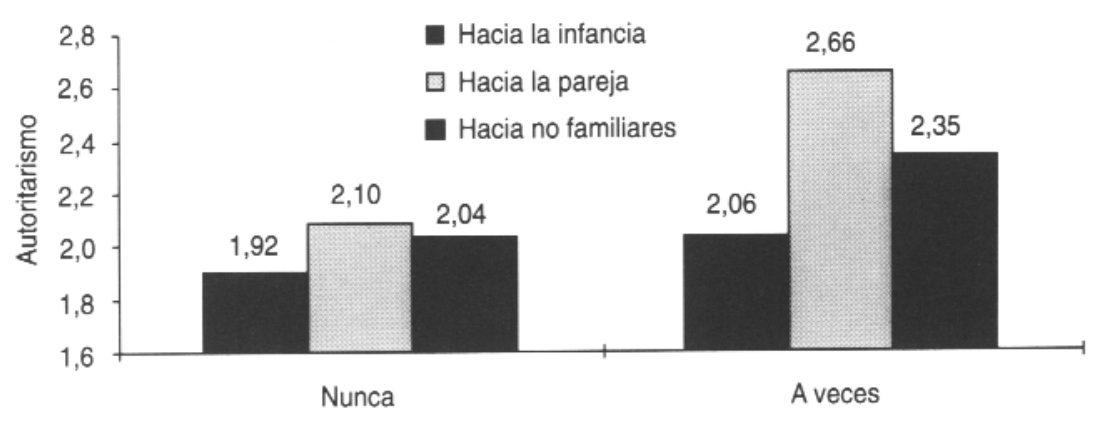

Ejercen conductas violentas 
"insultó a alguien" no permitió discriminar a las personas más o menos autoritarias (los valores que aparecen se refieren a los dos ítemes referidos a amenazar y a golpear). En los cuatro casos, las diferencias fueron también estadísticamente significativas. Los valores $P$ asociados con el estadístico de contraste fueron 0,041 para los niños; 0,002 , para la pareja, $\mathrm{y}<0,0005$, para la violencia hacia no familiares; siempre en el sentido previsto: los valores medios de autoritarismo fueron mayores en el grupo de personas que manifestaron ejercer la violencia que en el grupo de los que manifestaron no ejercerla.

De todas las características sociodemográficas analizadas, las que tuvieron una relación más clara con las actitudes autoritarias fueron, primero, el nivel educativo; como se observa en la figura 3, el autoritarismo aumenta a medida que desciende el nivel educativo de las personas $(P<0,0005)$. Segundo, el nivel socioeconómico, en el sentido de que las personas que viven en barrios con menos recursos tuvieron puntajes más altos en autoritarismo $(2,2)$ que las de niveles altos o medios (2) $(P=0,0022)$. Tercero, la situación laboral: las personas que no tenían trabajo tuvieron puntajes más altos $(2,23)$ que las que trabajaban $(1,48)(P=0,01)$. Cuarto, la orientación política: las personas que se identificaron con ideología de derecha $(2,34)$ eran más autoritarias que las de izquierda $(1,79)(P<$ $0,0005)$. Quinto, la edad: el autoritarismo es mayor en las personas de más edad, aunque con las dos excepciones que pueden observarse en la figura 4. Por último, no se observaron diferencias estadísticamente significativas en cuanto al nivel de autoritarismo entre varones y mujeres.

\section{DISCUSIÓN}

Aunque los resultados indican que los niveles medios de autoritarismo, de justificación de la violencia y de conductas violentas declarados por los entrevistados en este estudio son bajos (siempre por debajo de la media teórica), para interpretarlos hay que tener
FIGURA 3. Autoritarismo versus nivel educativo. Madrid, España, 1996

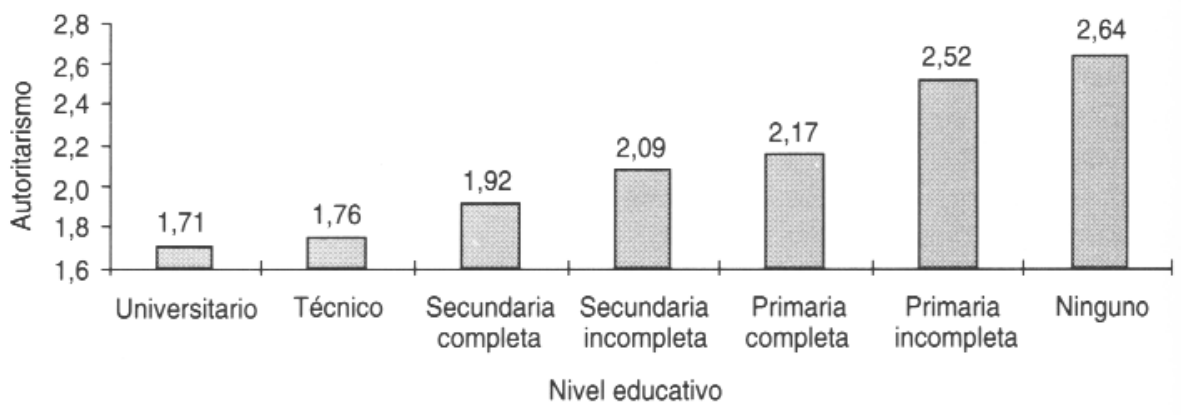

en cuenta el efecto de deseabilidad social y la gravedad de la mayor parte de las conductas expresadas en el cuestionario (muchas de ellas alusivas a matar, golpear con objetos duros, etc.). En Madrid hay una gran presión social en torno a los malos tratos; no solo existe cierto consenso en condenar moralmente a quienes los ejercen, sino que la mayor parte de las conductas expresadas en el cuestionario (a excepción de gritar e insultar) son punibles legalmente.

Independientemente de la gravedad del nivel de violencia ejercido, lo que se pone de manifiesto con los datos obtenidos es que determinado tipo de actitudes (las que se han denominado autoritarias), que aparentemente podrían no tener una relación directa con el hecho de justificar y ejercer la violencia hacia quienes tenemos más cerca de

nosotros, están íntimamente relacionadas con el ejercicio efectivo de la misma.

En el caso de la justificación de la violencia, parece existir una relación muy clara con el autoritarismo. La relación de la violencia ejercida con el autoritarismo se daba de forma clara en los casos de violencia hacia niños, pero hacia la pareja y los no familiares solo se daba cuando se aludía a manifestaciones graves de violencia (bofetadas, amenazas, golpes con objetos duros, etc.). Es probable que algunas conductas como gritar o insultar no sean entendidas por muchos madrileños como manifestaciones violentas. De hecho, en el proceso de recolección de datos muchos entrevistados manifestaban que en su lenguaje diario incluían insultos. En España, el tono de voz con el que habitualmente se reali-

\section{FIGURA 4. Autoritarismo versus edad. Madrid, España, 1996}

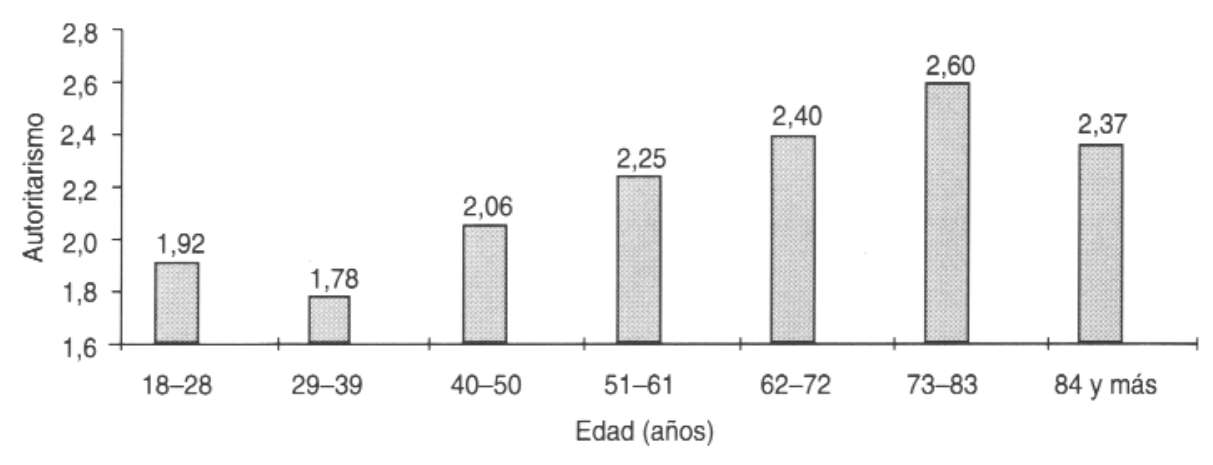


zan las interacciones suele ser más alto que el que se adopta en otras latitudes.

En la figura 4 se aprecia que la tendencia a observar valores más altos en autoritarismo a medida que aumenta la edad, no se cumple en las personas de 29 a 39 años (los menos autoritarios), ni en las más ancianas (84 a 94), que son menos autoritarias que las de 62 a 83 años. Caben dos interpretaciones de estos resultados: primero, la franja de edad menos autoritaria (29 a 39 años) parece coincidir con el período en el que las personas están más integradas socialmente (están criando a sus hijos, habitualmente tienen trabajo, son reconocidos socialmente, etc.), elementos que parecen contradecir la idea de aislamiento y temor que puede asociarse con el autoritarismo; los más ancianos serían menos autoritarios por un proceso de desvinculación con el mundo (27); en esa edad postrera no les preocuparía mucho la homogeneidad social ni confiarían demasiado en las autoridades. La segunda interpretación, más vinculada con la cultura española, sería que las personas de 29 a 39 años de edad se socializaron en un período político muy especial de nuestro país (la transición de la dictadura a la democracia), y que la generación de los mayores de 84 años vivió su infancia y adolescencia en el período previo a la guerra civil y el franquismo.

Entre la situación potencialmente violenta (conflictos, provocaciones, estrés, frustración, etc.) y la conducta violenta explícita media un conjunto de disposiciones (habilidades, actitudes, etc.) que da sentido a la acción y la hace más o menos posible. Las actitudes autoritarias estudiadas en el presente artículo son un componente más de esas disposiciones. Si los que tienden a sobrevalorar a los poderosos y a rechazar lo diferente justifican de forma más clara la violencia, e incluso la ejercen más, la aplicación parece clara: es preciso fomentar actitudes no autoritarias; esto es, actitudes críticas hacia quienes abusan del poder y de respeto por la presencia y la conducta de quienes son diferentes. Ahora bien, fomentar actitudes antiautoritarias no es suficiente para disminuir el nivel de violencia. De los datos obtenidos en el proyecto ACTIVA se podrán sacar numerosas pistas que deberían tenerse en cuenta: experiencias violentas vividas (malos tratos en la infancia, victimización, exposición a modelos violentos en la televisión, etc.), influencia de las habilidades autopercibidas, consumo de sustancias, posesión de armas, así como otros elementos que exceden los límites de esta investigación (estructura económica, organización política, etc.).

En diversos trabajos se ha analizado cómo el sistema social hace que los individuos participen de forma efectiva en actos violentos $(9,28)$. En estos estudios, al igual que en el citado de Adorno (14), se analiza el proceso de justificación de la violencia política, especialmente el proceso de participación en las guerras. Los datos expuestos anteriormente parecen ir más allá de la influencia del pensamiento autoritario en la política y permiten concluir que las personas socializadas en valores autoritarios tenderán a justificar y ejercer más la violencia, sea cual fuere el ámbito en que esta se desarrolle. El proceso parece ser siempre el mismo (29): se comienza con la polarización (identificación con el grupo de referencia), de ahí se pasa a la justificación de las actividades de estos grupos, y se termina con la acomodación funcional a las demandas recibidas, siempre que vayan en la línea de defender el endogrupo frente a las amenazas (reales o inventadas) del exogrupo. Romper esta cadena actitudinal no es sencillo. Las actitudes autoritarias se basan en la imagen de un mundo de orden sin espacio para las diferencias o el conflicto, un sueño demagógico que cuando se intenta convertir en realidad desemboca en la pesadilla de la violencia.

Si la conformación de las actitudes autoritarias no se entiende como rasgo inmutable de la personalidad, sino como resultado de las normas de raíz histórica en cada ámbito cultural, sería importante, una vez establecida la relación autoritarismo-disposición a la violencia, investigar los vínculos de la normatividad social y política con la conformación y cambio de las actitudes autoritarias. En este esfuerzo se debería atender a las demandas por una educación familiar y escolar capaz de transmitir normas sin utilizar procedimientos autoritarios; a la oposición a los distintos intentos de adoctrinamiento por medio de la propaganda explícita o la más sofisticada industria de la publicidad, y a la reflexión sobre nuevos fenómenos autoritarios que anulan el pensamiento crítico por procedimientos más sutiles como la imposición, a través de poderosos medios de comunicación, de formas aparentemente universales de entender e interpretar el mundo, las relaciones humanas y la corrección política.

Agradecimiento. Este estudio no hubiera sido posible sin la colaboración de la Consejería de Educación y Cultura de la Comunidad de Madrid, que financió el trabajo de campo de la investigación a través de la Dirección General de Investigación (proyecto 05I/020/96). Este artículo se basa en los datos del Estudio Multicéntrico sobre Actitudes y Normas Culturales frente a la Violencia (proyecto ACTIVA), realizado en ocho ciudades de América Latina y España bajo los auspicios y la coordinación de la Organización Panamericana de la Salud. 
1. Ayuntamiento de Madrid. Anuario estadístico municipal. Madrid: Artes Gráficas Municipales;1997.

2. Moreno Martín F. Explicaciones de la violencia: vías ciegas para la intervención. Energía, Carácter y Sociedad 1996;14:165-178.

3. Lorenz K. L'agression. Paris: Flammarion; 1969.

4. Wilson EO. Sociobiology. The new synthesis. Cambridge, Mass: The Belknap Press of Harvard University Press; 1975.

5. Genovés S. Expedición a la violencia. México, DF: Universidad Nacional Autónoma de México; 1991.

6. Berkowitz L. Aggression: its causes, consequences, and control. Philadelphia: Temple University Press; 1993.

7. Bandura A. Aggression: a social learning analysis. Englewood Cliffs, NJ: Prentice Hall; 1973.

8. Wood W, Wong FY, Chachere JG. Effects of media violence on viewers' aggression in unconstrained social interaction. Psychol Bull 1991;109:307-383.

9. Moreno Martín F. Identidad y violencia política. En: Younis JA, ed. Cultura, Psicología y Problemas Sociales. Las Palmas: Nogal Ediciones; 1995:151-164.
10. Moreno Martín F. Infancia y guerra en Centroamérica. San José: Editorial FLACSO; 1991.

11. Moreno Martín F, Jiménez F. La guerra: realidad y alternativas. Madrid: Editorial Complutense; 1992.

12. Milgram S. Obedience to authority. New York: Harper \& Row; 1974

13. Moreno Martín F. Reinserción de guerrilleros: ¿entrando en la casa del enemigo? Historia Crítica 1993;7:30-39.

14. Adorno T, Frenkel-Brunswik E, Levinson D, Sanford R. The authoritarian personality. New York: Harper; 1950.

15. Christie R, Jahoda M, eds. Studies in the scope and method of "The authoritarian personality". Glencoe, Ill: The Free Press; 1965.

16. Hyman HH, Sheatsley PB. The authoritarian personality, a methodological critique. En: Christie R, Jahoda M, eds. Studies in the scope and method of "The authoritarian personality". Glencoe, Ill.: The Free Press; 1965.

17. Billig M. Social psychology and intergroup relations. London: Academic Press; 1976.

18. Eysenck HJ. The psychology of politics. London: Routledge \& Kegan Paul; 1954.

19. Rokeach M. Generalized mental rigidity as factor in ethnocentrism. J Abnormal Soc Psychol 1948;43:259-278.
20. Rokeach M. The open and closed mind. New York: Basic Books; 1960

21. Pettigrew TF. Personality and sociocultural factors in intergroup attitudes: a crossnational comparison. J Conflict Resolution 1958; 2:29-42

22. Fishbein M, Ajzen I. Belief, attitude, intention and behavior: an introduction to theory and research. Reading, Mass.: Addison-Wesley; 1975.

23. Brown R. Social psychology. New York: MacMillan; 1965

24. Rodríguez Osuna J. Métodos de muestreo. Madrid: Centro de Investigaciones Sociológicas; 1991.

25. Likert R. Una técnica para medir actitudes. En: Summers GF. Medición de actitudes. México: Trillas; 1976.

26. Moreno Martín F. La polarización, el pretendido mal. Interacción Social 1993;3:117-132.

27. Avia MD, Sánchez ML. Personalidad: aspectos cognitivos y sociales. Madrid: Pirámide; 1995.

28. Withey S, Katz D. The social psychology of human conflict. En: McNeill E, ed. The nature of human conflict. New Jersey: Prentice Hall; 1965:92-125.

29. Moreno Martín F. La socialización bélica. Estudio empírico en Centroamérica y España. Madrid: Editorial Complutense; 1991.

ABSTRACT One of the objectives of the Multicentric Study on Cultural Norms and Attitudes Toward Violence (Estudio Multicéntrico sobre Actitudes y Normas Culturales frente a la Violencia, the ACTIVA project) is to analyze the relationship between those attitudes that foster violence and the violent behaviors themselves. This article examines the relationship between attitudes and two components of violence: its justification and its actual occurrence. Data were collected in Madrid between October and December 1996 from a representative sample of 1219 people, who were interviewed at home with the common questionnaire used for the ACTIVA project, with some additional questions. Overall, the sample respondents scored low on the authoritarianism scale. Persons who most strongly justified the use of violence scored higher on authoritarianism, along with those who customarily displayed a higher level of aggression. Attitudes that were more strongly authoritarian were found in low-income neighborhoods, in people who were not part of the workforce, in people with less education, and in those persons who described themselves as having a right-wing ideology. If people who are more authoritarian justify and practice violence more than others, it becomes necessary to encourage criticism of those who abuse their power and tolerance toward differences, in order to prevent such behaviors without disregarding the influence of the social variables previously mentioned. 